\title{
Real Number
}

National Cancer Institute

\section{Source}

National Cancer Institute. Real Number. NCI Thesaurus. Code C62443.

Any of the rational or irrational numbers. 